\title{
More set-theory around the weak Freese-Nation property
}

\author{
by \\ Sakaé F u chino (Berlin and Kitami) and \\ Lajos S ou kup (Budapest)
}

\begin{abstract}
We introduce a very weak version of the square principle which may hold even under failure of the generalized continuum hypothesis. Under this weak square principle, we give a new characterization (Theorem 10) of partial orderings with $\kappa$-Freese-Nation property (see below for the definition). The characterization is not a ZFC theorem: assuming Chang's Conjecture for $\aleph_{\omega}$, we can find a counter-example to the characterization (Theorem 12). We then show that, in the model obtained by adding Cohen reals, a lot of ccc complete Boolean algebras of cardinality $\leq \lambda$ have the $\aleph_{1}$-Freese-Nation property provided that $\mu^{\aleph_{0}}=\mu$ holds for every regular uncountable $\mu<\lambda$ and the very weak square principle holds for each cardinal $\aleph_{0}<\mu<\lambda$ of cofinality $\omega$ (Theorem 15). Finally, we prove that there is no $\aleph_{2}$-Lusin gap if $\mathcal{P}(\omega)$ has the $\aleph_{1}$-Freese-Nation property (Theorem 17).
\end{abstract}

1. Introduction. For a regular $\kappa$, a partial ordering $P$ is said to have the $\kappa$-Freese-Nation property (the $\kappa$-FN for short) if there is a mapping $\left(\kappa-F N\right.$ mapping) $f: P \rightarrow[P]^{<\kappa}$ such that for any $p, q \in P$ if $p \leq q$ then there is $r \in f(p) \cap f(q)$ such that $p \leq r \leq q$.

Freese and Nation [5] used the $\aleph_{0}-\mathrm{FN}$ in a characterization of projective lattices and asked if this property alone already characterizes the projectiveness. L. Heindorf gave a negative answer to the question showing that the Boolean algebras with the $\aleph_{0}-\mathrm{FN}$ are exactly those which are openly generated. It is known that the class of openly generated Boolean algebras contains projective Boolean algebras as a proper subclass (see [8] — openly generated Boolean algebras are called "rc-filtered" there). Heindorf and Shapiro [8] then studied the $\aleph_{1}$-FN which they called the weak Freese-Nation property and proved some elementary properties of the Boolean algebras with this property. Partial orderings with the $\kappa$-FN for arbitrary regular $\kappa$ were

1991 Mathematics Subject Classification: 03E05, 03E35, 03E55, 06A06, 06E05.

The second author was supported by the Hungarian National Foundation for Scientific Research grant no. 16391. 
further studied in Fuchino, Koppelberg and Shelah [6]. Koppelberg [10] gives some nice applications of the $\aleph_{1}$-FN.

In the following we shall quote some elementary facts from [6] which we need later. First of all, it can be readily seen that every small partial ordering has the $\kappa$-FN:

Lemma 1 ([6]). Every partial ordering $P$ of cardinality $\leq \kappa$ has the $\kappa-F N$.

For a partial ordering $P$ and a subordering $Q \subseteq P$, we say that $Q$ is a $\kappa$-subordering of $P$ and write $Q \leq_{\kappa} P$ if, for every $p \in P$, the set $\{q \in Q$ : $q \leq p\}$ has a cofinal subset of cardinality $<\kappa$ and the set $\{q \in Q: q \geq p\}$ has a coinitial subset of cardinality $<\kappa$.

Lemma $2([6])$. Suppose that $\delta$ is a limit ordinal and $\left(P_{\alpha}\right)_{\alpha \leq \delta}$ a continuously increasing chain of partial orderings such that $P_{\alpha} \leq_{\kappa} P_{\delta}$ for all $\alpha<\delta$. If $P_{\alpha}$ has the $\kappa-F N$ for every $\alpha<\delta$, then $P_{\delta}$ also has the $\kappa-F N$.

For application of Lemma 2, it is enough to have $P_{\alpha} \leq_{\kappa} P_{\delta}$ and the $\kappa$-FN of $P_{\alpha}$ for every $\alpha<\delta$ such that either $\alpha$ is a successor or of cofinality $\geq \kappa$ : $P_{\alpha} \leq_{\kappa} P_{\delta}$ for $\alpha<\delta$ of cofinality $<\kappa$ follows from this since such a $P_{\alpha}$ can be represented as the union of $<\kappa$ many $\kappa$-suborderings of $P_{\delta}$. Hence by inductive application of Lemma 2, we can show that $P_{\alpha}$ satisfies the $\kappa$-FN for every $\alpha \leq \delta$. Similarly, if $\delta$ is a cardinal $>\kappa$, then it is enough to have $P_{\alpha}<_{\kappa} P_{\delta}$ and the $\kappa$-FN of $P_{\alpha}$ for every limit $\alpha<\delta$ of cofinality $\geq \kappa$.

Proposition 3 ([6]). For a regular $\kappa$ and a partial ordering $P$, the following are equivalent:

(1) $P$ has the $\kappa-F N$;

(2) For some, or equivalently, any sufficiently large $\chi$, if $M \prec \mathcal{H}_{\chi}=$ $\left(\mathcal{H}_{\chi}, \in\right)$ is such that $P \in M, \kappa \subseteq M$ and $|M|=\kappa$ then $P \cap M \leq_{\kappa} P$;

(3) $\left\{C \in[P]^{\kappa}: C \leq_{\kappa} P\right\}$ contains a club set.

Though Proposition 3(2) is quite useful to show that a partial ordering has the $\kappa$-FN, sometimes it is quite difficult to check Proposition 3(2) as in the case of the $\aleph_{1}-\mathrm{FN}$ of $P(\omega)$ or $[\kappa]^{<\omega}$ : in these cases it is independent if Proposition 3(2) holds. With applications like Corollary 11 in mind, we could think of another possible variant of Proposition 3(2) in terms of the following weakening of the notion of internal approachability from [4]: for a regular $\kappa$ and a sufficiently large $\chi$, we shall call an elementary submodel $M$ of $\mathcal{H}_{\chi} V_{\kappa}$-like if either $\kappa=\aleph_{0}$ and $M$ is countable, or there is an increasing sequence $\left(M_{\alpha}\right)_{\alpha<\kappa}$ of elementary submodels of $M$ of cardinality less than $\kappa$ such that $M_{\alpha} \in M_{\alpha+1}$ for all $\alpha<\kappa$ and $M=\bigcup_{\alpha<\kappa} M_{\alpha}$. 
In [6], a characterization of the $\kappa$-FN using $V_{\kappa}$-like elementary submodels in place of elementary submodels in Proposition 3(2) was discussed. Unfortunately, it appeared that some consequences of $\neg 0^{\#}$ are necessary for the characterization (see "Added in Proof" in [6]). In this paper, we introduce a weakening of the very weak square principle from [3] - the principle $\square_{\kappa, \mu}^{* * *}$. In Section 2 we show the equivalence of $\square_{\kappa, \mu}^{* * *}$ with the existence of a matrix $\left(M_{\alpha, \beta}\right)_{\alpha<\mu^{+}, \beta<\operatorname{cf}(\mu)}$-which we called a (weak) $(\kappa, \mu)$-dominating matrix - of elementary submodels of $\mathcal{H}(\chi)$ for sufficiently large $\chi$ with certain properties. This fact is used in Section 3 to show that $\square_{\kappa, \lambda}^{* * *}$ together with a very weak version of the Singular Cardinals Hypothesis yields the characterization of partial orderings with the $\kappa$-FN in terms of $V_{\kappa}$-like elementary submodels (Theorem 10). ZFC or even ZFC + GCH is not enough for this characterization: in Section 4, we show that, under Chang's Conjecture for $\aleph_{\omega}$, there is a counter-example to the characterization. Together with Theorem 10, this counter-example also shows that $\square_{\aleph_{1}, \aleph_{\omega}}^{* * *}$ is not a theorem in ZFC + GCH.

One of the most natural questions concerning the $\kappa$-FN is whether $(\mathcal{P}(\omega)$, $\subseteq)$ has the $\aleph_{1}$-FN. It is easy to see that $(\mathcal{P}(\omega), \subseteq)$ has the $\aleph_{1}$-FN iff $\left(\mathcal{P}(\omega) /\right.$ fin, $\left.\subseteq^{*}\right)$ does (see [6]). See also Koppelberg [10] for some consequences of the $\aleph_{1}$-FN of $\mathcal{P}(\omega) /$ fin. By Lemma $1, \mathcal{P}(\omega)$ has the $\aleph_{1}$-FN under $\mathrm{CH}$. In Section 5, we show that $\mathcal{P}(\omega)$ and a lot of other ccc complete Boolean algebras still have the $\aleph_{1}-\mathrm{FN}$ in a model obtained by adding an arbitrary number of Cohen reals to a model of, say, $V=L$. On the other hand, it can happen very easily that $\mathcal{P}(\omega)$ does not have the $\aleph_{1}-\mathrm{FN}$. In [6], it was shown that this is the case when $\mathbf{b}>\aleph_{1}$ or, more generally, if there is a $\subseteq^{*}$-sequence of elements of $\mathcal{P}(\omega)$ of order-type $\geq \omega_{2}$. In Section 6 , we show that the existence of an $\aleph_{2}$-Lusin gap can be another reason for failure of the $\aleph_{1}$-FN. At the moment the authors do not know if there are yet other reasons for failure of the $\aleph_{1}-\mathrm{FN}$ of $\mathcal{P}(\omega)$ :

Problem 1. Suppose that $\mathcal{P}(\omega)$ does not have any increasing chain of length $\geq \aleph_{2}$ with respect to $\subseteq^{*}$ and that there is no $\aleph_{2}$-Lusin gap. Does it follow that $\mathcal{P}(\omega)$ has the $\aleph_{1}-F N$ ?

Our notation is fairly standard. The following are possible deviations from the standard: for $C \subseteq \kappa$, we denote by $(C)^{\prime}$ the set of limit points of $C$ other than $\kappa$. For an ordinal $\alpha, \operatorname{Lim}(\alpha)=\{\beta<\alpha: \beta$ is a limit ordinal $\}$. For a partial ordering $P, \operatorname{cf}(P)=\min \{|X|: X \subseteq P, X$ is cofinal in $P\} \cdot[\lambda]^{<\kappa}=$ $\{X \subseteq \lambda:|X|<\kappa\}$ is often seen as the partial ordering $\left([\lambda]^{<\kappa}, \subseteq\right)$. If $Q$ is a subordering of a partial ordering $P$ and $p \in P$ then $Q \uparrow p=\{q \in Q: q \geq p\}$ and $Q\lceil p=\{q \in Q: q \leq p\}$. Let $I$ be an infinite set. Adopting the notation of [12], we denote by $\operatorname{Fn}(I, 2)$ the standard partial ordering for adding $|I|$ Cohen reals, i.e. $\operatorname{Fn}(I, 2)$ is the partial ordering $\{p: p$ is a mapping with $\operatorname{dom}(p) \in$ $[I]^{<\aleph_{0}}$ and range $\left.(p) \subseteq 2\right\}$ with the inverse inclusion. 
2. Very weak square and dominating matrix. For a cardinal $\mu$, the weak square principle for $\mu$ (notation: $\square_{\mu}^{*}$ ) is the statement: there is a sequence $\left(\mathcal{C}_{\alpha}\right)_{\alpha \in \operatorname{Lim}\left(\mu^{+}\right)}$such that for every $\alpha \in \operatorname{Lim}\left(\mu^{+}\right)$,

(w1) $\mathcal{C}_{\alpha} \subseteq \mathcal{P}(\alpha)$ and $\left|\mathcal{C}_{\alpha}\right| \leq \mu$;

(w2) every $C \in \mathcal{C}_{\alpha}$ is a club in $\alpha$ and if $\operatorname{cf}(\alpha)<\mu$ then $\operatorname{otp}(C)<\mu$;

(w3) for every $C \in \mathcal{C}_{\alpha}$ and $\delta \in(C)^{\prime}$, we have $C \cap \delta \in \mathcal{C}_{\delta}$.

Clearly we have $\square_{\mu} \rightarrow \square_{\mu}^{*}$. Jensen [9] proved that $\square_{\mu}^{*}$ is equivalent to the existence of a special Aronszajn tree on $\mu^{+}$. Ben-David and Magidor [2] showed that the weak square principle for a singular $\mu$ is actually weaker than the square principle: they constructed a model of $\square_{\aleph_{\omega}}^{*}$ and $\neg \square_{\aleph_{\omega}}$ starting from a model with a supercompact cardinal.

Foreman and Magidor considered in [3] the following principle which is, e.g. under $\mathrm{GCH}$, a weakening of the $\square^{*}$ principle: for a cardinal $\mu$, the very weak square principle for $\mu$ holds if there is a sequence $\left(C_{\alpha}\right)_{\alpha<\mu^{+}}$and a club $D \subseteq \mu^{+}$such that for every $\alpha \in D$,

(v1) $C_{\alpha} \subseteq \alpha, C_{\alpha}$ is unbounded in $\alpha$;

(v2) for all bounded $x \in\left[C_{\alpha}\right]^{<\omega_{1}}$, there is $\beta<\alpha$ such that $x=C_{\beta}$.

In this paper, we shall use the following yet weaker variant of the very weak square principle. For a regular cardinal $\kappa$ and $\mu>\kappa$, let $\square_{\kappa, \mu}^{* * *}$ be the following assertion: there exists a sequence $\left(C_{\alpha}\right)_{\alpha<\mu^{+}}$and a club set $D \subseteq \mu^{+}$ such that for $\alpha \in D$ with $\operatorname{cf}(\alpha) \geq \kappa$,

(y1) $C_{\alpha} \subseteq \alpha, C_{\alpha}$ is unbounded in $\alpha$;

(y2) $[\alpha]^{<\kappa} \cap\left\{C_{\alpha^{\prime}}: \alpha^{\prime}<\alpha\right\}$ dominates $\left[C_{\alpha}\right]^{<\kappa}$ (with respect to $\subseteq$ ).

Since (y2) remains valid when $C_{\alpha}$ 's for $\alpha \in D$ are shrunk, we may replace (y1) by

(y1') $C_{\alpha} \subseteq \alpha, C_{\alpha}$ is unbounded in $\alpha$ and $\operatorname{otp}\left(C_{\alpha}\right)=\operatorname{cf}(\alpha)$.

A corresponding remark holds also for the sequence of the very weak square principle.

Lemma 4. (a) The very weak square principle for $\mu$ implies $\square_{\omega_{1}, \mu}^{* * *}$.

(b) For a singular cardinal $\mu$ and a regular $\kappa$ such that $\operatorname{cf}\left([\lambda]^{<\kappa}, \subseteq\right) \leq \mu$ for every $\lambda<\mu, \square_{\mu}^{*}$ implies $\square_{\kappa, \mu}^{* * *}$.

Pr o of. (a) is clear. For (b), let $\left(\mathcal{C}_{\alpha}\right)_{\alpha \in \operatorname{Lim}\left(\mu^{+}\right)}$be a weak square sequence. Let $\mathcal{C}_{\alpha}=\left\{C_{\alpha, \beta}: \beta<\mu\right\}$ for every $\alpha \in \operatorname{Lim}\left(\mu^{+}\right)$. By shrinking $C_{\alpha, \beta}$ 's if necessary, we may assume that $\left|C_{\alpha, \beta}\right|<\mu$ for every $\alpha \in \operatorname{Lim}\left(\mu^{+}\right)$and $\beta<\mu$. Note that we need here the assumption that $\mu$ be singular. For $\alpha \in \operatorname{Lim}\left(\mu^{+}\right)$ and $\beta<\mu$, let $X_{\alpha, \beta}$ be a cofinal subset of $\left[C_{\alpha, \beta}\right]^{<\kappa}$ of cardinality $\leq \mu$ and let $\left\{C_{\alpha}: \alpha \in \mu^{+} \backslash \operatorname{Lim}\left(\mu^{+}\right)\right\}$be an enumeration of $\bigcup\left\{X_{\alpha, \beta}: \alpha \in \operatorname{Lim}\left(\mu^{+}\right)\right.$, $\beta<\mu\}$. For each $\alpha \in \operatorname{Lim}\left(\mu^{+}\right)$, let $C_{\alpha}=C_{\alpha, 0}$. Let $F: \mu^{+} \rightarrow \mu^{+}$be defined 
by $F(\xi)=\min \left\{\gamma<\mu^{+}: X_{\xi, \beta} \subseteq\left\{C_{\alpha}: \alpha<\gamma\right\}\right.$ for every $\left.\beta<\mu\right\}$. Let $D \subseteq \mu^{+}$be a club set closed with respect to $F$. Then $\left(C_{\alpha}\right)_{\alpha<\mu^{+}}$and $D$ are as in the definition of $\square_{\kappa, \mu}^{* * *}$. To see that (y2) is satisfied, let $\alpha \in D$ be such that $\operatorname{cf}(\alpha) \geq \kappa$ and $x \in\left[C_{\alpha}\right]^{<\kappa}$. By definition we have $C_{\alpha}=C_{\alpha, 0}$. Hence there are $\alpha^{\prime} \in \alpha \cap \operatorname{Lim}\left(\mu^{+}\right)$and $\beta<\lambda$ such that $x \in\left[C_{\alpha^{\prime}, \beta}\right]^{<\kappa}$. Since $\alpha$ is closed with respect to $F$, there is some $\gamma<\alpha$ such that $x \subseteq C_{\gamma} \in\left[C_{\alpha^{\prime}, \beta}\right]^{<\kappa}$. - Lemma 4

$\square_{\kappa, \mu}^{* * *}$ has some influence on cardinal arithmetic of cardinals below $\mu$ :

Lemma 5. Suppose that $\kappa$ is regular and $\mu$ is such that $\operatorname{cf}(\mu)<\kappa$. If $\square_{\kappa, \mu}^{* * *}$ holds, then $\operatorname{cf}\left([\lambda]^{<\kappa}, \subseteq\right)<\mu$ for every $\lambda<\mu$.

Pr o of. Let $\left(C_{\alpha}\right)_{\alpha<\mu^{+}}$and $D \subseteq \mu^{+}$be witnesses of $\square_{\kappa, \mu}^{* * *}$. For $\lambda<\mu$, let $\delta \in D$ be such that $\operatorname{cf}(\delta) \geq \lambda+\kappa$. Then $\left\{C_{\alpha}: \alpha<\delta\right\} \cap[\delta]^{<\kappa}$ is cofinal in $\left[C_{\delta}\right]^{<\kappa}$. Since $|\delta| \leq \mu$ it follows that $\operatorname{cf}\left(\left[C_{\delta}\right]^{<\kappa}, \subseteq\right) \leq \mu$. As the order type of $C_{\delta}$ is at least $\lambda$, it follows that $\nu=\operatorname{cf}\left([\lambda]^{<\kappa}, \subseteq\right) \leq \mu$. But $\operatorname{cf}(\nu) \geq \kappa$. Hence $\nu<\mu$. $\mathbf{I e m m a}_{5}$

Suppose now that $\kappa$ is a regular cardinal and $\mu>\kappa$ is such that $\operatorname{cf}(\mu)<\kappa$. Let $\mu^{*}=\operatorname{cf}(\mu)$. For a sufficiently large $\chi$ and $x \in \mathcal{H}(\chi)$, let us call a sequence $\left(M_{\alpha, \beta}\right)_{\alpha<\mu^{+}, \beta<\mu^{*}}$ a $(\kappa, \mu)$-dominating matrix over $x$ - or just a dominating matrix over $x$ if it is clear from the context which $\kappa$ and $\mu$ are meant-if the following conditions hold:

(j1) $M_{\alpha, \beta} \prec \mathcal{H}(\chi), x \in M_{\alpha, \beta}, \kappa+1 \subseteq M_{\alpha, \beta}$ and $\left|M_{\alpha, \beta}\right|<\mu$ for all $\alpha<\mu^{+}$and $\beta<\mu^{*}$;

(j2) $\left(M_{\alpha, \beta}\right)_{\beta<\mu^{*}}$ is an increasing sequence for each $\alpha<\mu^{+}$;

(j3) if $\alpha<\mu^{+}$is such that $\operatorname{cf}(\alpha) \geq \kappa$, then there is $\beta^{*}<\mu^{*}$ such that, for every $\beta^{*} \leq \beta<\mu^{*},\left[M_{\alpha, \beta}\right]^{<\kappa} \cap M_{\alpha, \beta}$ is cofinal in $\left(\left[M_{\alpha, \beta}\right]^{<\kappa}, \subseteq\right)$. $\mathcal{H}(\chi)$.

For $\alpha<\mu^{+}$, let $M_{\alpha}=\bigcup_{\beta<\mu^{*}} M_{\alpha, \beta}$. By (j1) and (j2), we have $M_{\alpha} \prec$

(j4) $\left(M_{\alpha}\right)_{\alpha<\mu^{+}}$is continuously increasing and $\mu^{+} \subseteq \bigcup_{\alpha<\mu^{+}} M_{\alpha}$.

Foreman and Magidor [3] called a sequence of subsets of $\mu^{+}$having some properties similar to those of the sequence $\left(\mu^{+} \cap M_{\alpha, \beta}\right)_{\alpha<\mu^{+}, \beta<\mu^{*}}$ for $\left(M_{\alpha, \beta}\right)_{\alpha<\mu^{+}, \beta<\mu^{*}}$ as above a Jensen matrix. Our definition of dominating matrices and some ideas in the proofs in this section are inspired by their paper. Note that, in the case of $2^{<\kappa}=\kappa,(\mathrm{j} 3)$ can be replaced by the following seemingly stronger property:

$\left(\mathrm{j} 3^{\prime}\right)$ if $\alpha<\mu^{+}$is such that $\operatorname{cf}(\alpha) \geq \kappa$, then there is $\beta^{*}<\mu^{*}$ such that, for every $\beta^{*} \leq \beta<\mu^{*},\left[M_{\alpha, \beta}\right]^{<\kappa} \subseteq M_{\alpha, \beta}$.

This is simply because of the following observation: 
Lemma 6. Suppose that $2^{<\kappa}=\kappa$ and $M$ is an elementary submodel of $\mathcal{H}(\chi)$ for some sufficiently large $\chi$ and $\kappa \subseteq M$. If $[M]^{<\kappa} \cap M$ is cofinal in $[M]^{<\kappa}$, then $[M]^{<\kappa} \subseteq M$.

Proof. Let $x \in[M]^{<\kappa}$. We show that $x \in M$. By assumption there is $y \in[M]^{<\kappa} \cap M$ such that $x \subseteq y$. Let $\eta=|\mathcal{P}(y)|$. Then $\eta \in M$ and $\eta \leq \kappa$. Let $\left(y_{\alpha}\right)_{\alpha<\eta} \in M$ be an enumeration of $\mathcal{P}(y)$. Then there is an $\alpha_{0}<\eta$ such that $x=y_{\alpha_{0}}$. But since $\kappa \subseteq M$, we have $\alpha_{0} \in M$ and $y_{\alpha_{0}} \in M$ as well. Lemma 6

Note also that, if $M \prec \mathcal{H}(\chi)$ is $V_{\kappa}$-like, then $[M]^{<\kappa} \cap M$ is cofinal in $[M]^{<\kappa}$. Hence, under $2^{<\kappa}=\kappa, M \prec \mathcal{H}(\chi)$ is $V_{\kappa}$-like if and only if $|M|=\kappa$ and $[M]^{<\kappa} \subseteq M$.

In the following theorem, we show that $\square_{\kappa, \mu}^{* * *}$ together with a very weak version of the Singular Cardinals Hypothesis below $\mu$ implies the existence of a dominating matrix:

THEOREM 7. Suppose that $\kappa$ is a regular cardinal and $\mu>\kappa$ is such that $\operatorname{cf}(\mu)<\kappa$. If $\operatorname{cf}\left([\lambda]^{<\kappa}, \subseteq\right)=\lambda$ for cofinally many $\lambda<\mu$ and $\square_{\kappa, \mu}^{* * *}$ holds, then, for any sufficiently large $\chi$ and $x \in \mathcal{H}(\chi)$, there is a $(\kappa, \mu)$-dominating matrix over $x$.

Proof. Let $\mu^{*}=\operatorname{cf}(\mu)$ and $\left(\mu_{\beta}\right)_{\beta<\mu^{*}}$ be an increasing sequence of cardinals below $\mu$ such that $\mu_{0}>\mu^{*}, \sup \left\{\mu_{\beta}: \beta<\mu^{*}\right\}=\mu$ and $\operatorname{cf}\left(\left[\mu_{\beta}\right]^{<\kappa}, \subseteq\right)$ $=\mu_{\beta}$ for every $\beta<\mu^{*}$. Let $\left(C_{\alpha}\right)_{\alpha \in \mu^{+}}$and $D \subseteq \mu^{+}$be as in the definition of $\square_{\kappa, \mu}^{* * *}$. Without loss of generality, we may assume that $\left|C_{\alpha}\right| \leq \operatorname{cf}(\alpha)$ for all $\alpha<\mu^{+}$. We may also assume that $\alpha>\mu$ for every $\alpha \in D$.

In the following, we fix a well-ordering $\unlhd$ on $\mathcal{H}(\chi)$ and, when we talk about $\mathcal{H}(\chi)$ as a structure, we mean $\mathcal{H}(\chi)=(\mathcal{H}(\chi), \in, \unlhd) . X \subseteq \mathcal{H}(\chi)$ as a substructure of $\mathcal{H}(\chi)$ is thus the structure $\left(X, \in \cap X^{2}, \unlhd \cap X^{2}\right)$ - for notational simplicity we shall denote such a structure simply by $(X, \in, \unlhd)$.

Let $N \in \mathcal{H}(\chi)$ be an elementary substructure of $\mathcal{H}(\chi)$ such that $N$ contains everything needed below - in particular, we assume that $\mu^{+} \subseteq N$ and $x,\left(C_{\alpha}\right)_{\alpha<\mu^{+}}, D,\left(\mu_{\alpha}\right)_{\alpha<\mu^{*}} \in N$. Let $\left(N_{\xi}\right)_{\xi<\kappa}$ be an increasing sequence of elementary submodels of $\mathcal{H}(\chi)$ such that

(0) $N_{0}=N$;

(1) $N_{\xi} \in \mathcal{H}(\chi)$ for every $\xi<\kappa$ and

(2) $\left(N_{\eta}\right)_{\eta \leq \xi} \in N_{\xi+1}$ for every $\xi<\kappa$.

Now, for each $\xi<\kappa$, let

$$
\mathcal{N}_{\xi}=\left(N_{\xi}, \in, \unlhd, R_{\xi}, \kappa, \mu, \mu^{*}, D,\left(C_{\alpha}\right)_{\alpha<\mu^{+}},\left(\mu_{\alpha}\right)_{\alpha<\mu^{*}}, x, \eta\right)_{\eta<\xi}
$$

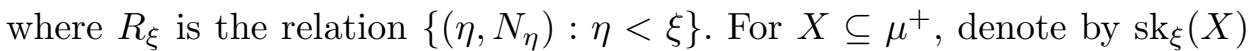
the Skolem-hull of $X$ with respect to the built-in Skolem functions of $\mathcal{N}_{\xi}$ (induced from $\unlhd$ ). For $\xi<\xi^{\prime}<\kappa, \mathcal{N}_{\xi}$ is an element of $\mathcal{N}_{\xi^{\prime}}$ by (2) and the Skolem functions of $\mathcal{N}_{\xi}$ are also elements of $\mathcal{N}_{\xi^{\prime}}$. In particular, we have 
$\operatorname{sk}_{\xi}(X) \subseteq \operatorname{sk}_{\xi^{\prime}}(X)$. It follows that $\operatorname{sk}(X)=\bigcup_{\xi<\kappa} \operatorname{sk}_{\xi}(X)$ is an elementary submodel of $\mathcal{H}(\chi)$. Note also that, if $X \subseteq \mu^{+}$is an element of $\operatorname{sk}_{\xi^{\prime}}(Y)$ then, since $\operatorname{sk}_{\xi}(X)$ is definable in $\operatorname{sk}_{\xi^{\prime}}(Y)$, we have $\operatorname{sk}_{\xi}(X) \in \operatorname{sk}_{\xi^{\prime}}(Y)$.

For the proof of the theorem, it is clearly enough to construct $M_{\alpha, \beta}$ with (j1)-(j4) for every $\alpha$ in the club set $D$ and for every $\beta<\mu^{*}$. Let

$$
M_{\alpha, \beta}=\operatorname{sk}\left(\mu_{\beta} \cup C_{\alpha}\right)
$$

for $\alpha \in D$ and $\beta<\mu^{*}$. We show that $\left(M_{\alpha, \beta}\right)_{\alpha \in D, \beta<\mu^{*}}$ is as desired. It is clear that (j1) and (j2) hold. We need the following claim to show the other properties:

Claim 7.1. $M_{\alpha}=\operatorname{sk}(\alpha)$ for every $\alpha \in D$.

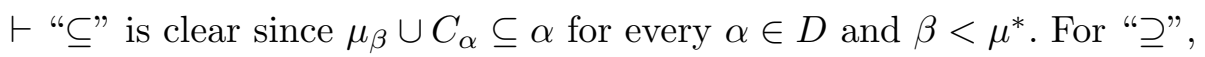
it is enough to show that $\alpha \subseteq M_{\alpha}$. Let $\gamma<\alpha$. By (y1), there is $\gamma_{1} \in C_{\alpha}$ such that $\gamma<\gamma_{1}$. Let $f \in M_{\alpha, 0}$ be a surjection from $\mu$ to $\gamma_{1}$, and let $\delta<\mu$ be such that $f(\delta)=\gamma$. Then $\gamma=f(\delta) \in M_{\alpha, \beta^{*}} \subseteq M_{\alpha}$ for $\beta^{*}<\mu^{*}$ such that $\delta<\mu_{\beta^{*}} \dashv_{\text {Claim } 7.1}$

For (j3), suppose that $\alpha \in D$ and $\operatorname{cf}(\alpha) \geq \kappa$. Let $\beta^{*}$ be such that $\left|C_{\alpha}\right|<$ $\mu_{\beta^{*}}$ and $[\alpha]^{<\kappa} \cap\left\{C_{\alpha^{\prime}}: \alpha^{\prime}<\alpha, \alpha^{\prime} \in M_{\alpha, \beta^{*}}\right\}$ dominates $\left[C_{\alpha}\right]^{<\kappa}$. The last property is possible by (y2), Claim 7.1 and $\mu^{*}<\kappa$. We show that this $\beta^{*}$ is as needed in $(\mathrm{j} 3)$. Let $\beta<\mu^{*}$ be such that $\beta^{*} \leq \beta$ and suppose that $x \in\left[M_{\alpha, \beta}\right]^{<\kappa}$. Then there are $u \in\left[\mu_{\beta}\right]^{<\kappa}$ and $v \in\left[C_{\alpha}\right]^{<\kappa}$ such that $x \subseteq \operatorname{sk}(u \cup v)$. Since $\mu_{\beta} \in M_{\alpha, \beta}$ and $\operatorname{cf}\left(\left[\mu_{\beta}\right]^{<\kappa}, \subseteq\right)=\mu_{\beta}$, there is $X \in M_{\alpha, \beta}$ such that $X \subseteq\left[\mu_{\beta}\right]^{<\kappa},|X|=\mu_{\beta}$ and $X$ is cofinal in $\left(\left[\mu_{\beta}\right]^{<\kappa}, \subseteq\right)$. Since $\mu_{\beta} \subseteq M_{\alpha, \beta}$, it follows that $X \subseteq M_{\alpha, \beta}$. Hence there is $u^{\prime} \in M_{\alpha, \beta} \cap\left[\mu_{\beta}\right]^{<\kappa}$ such that $u \subseteq u^{\prime}$. On the other hand, by definition of $\beta^{*}, \operatorname{cf}(\alpha) \geq \kappa$, there is $\alpha^{\prime} \in \alpha \cap M_{\alpha, \beta}$ such that $C_{\alpha^{\prime}} \in[\alpha]^{<\kappa}$ and $v \subseteq C_{\alpha^{\prime}}$. Thus, $x \subseteq \operatorname{sk}(u \cup v) \subseteq$ $\operatorname{sk}\left(u^{\prime} \cup C_{\alpha^{\prime}}\right)$. By regularity of $\kappa$, there is $\xi<\kappa$ such that $x \subseteq \operatorname{sk}_{\xi}\left(u^{\prime} \cup C_{\alpha^{\prime}}\right)$. But $\operatorname{sk}_{\xi}\left(u^{\prime} \cup C_{\alpha^{\prime}}\right) \in M_{\alpha, \beta}$ and $\left|\operatorname{sk}_{\xi}\left(u^{\prime} \cup C_{\alpha^{\prime}}\right)\right|<\kappa$.

(j4) follows immediately from Claim 7.1. - Theorem 7

Note that, in the proof above, the sequence $\left(M_{\alpha, \beta}\right)_{\alpha<\mu^{+}, \beta<\mu^{*}}$ satisfies also:

(j5) for $\alpha<\alpha^{\prime}<\mu^{+}$and $\beta<\mu^{*}$, there is $\beta^{\prime}<\mu^{*}$ such that $M_{\alpha, \beta} \subseteq$ $M_{\alpha^{\prime}, \beta^{\prime}}$.

[Suppose that $\alpha, \alpha^{\prime} \in D$ are such that $\alpha<\alpha^{\prime}$ and $\beta<\mu^{*}$. By Claim 7.1, there is $\beta^{\prime}<\mu^{*}$ such that $\beta<\beta^{\prime}, \alpha \in M_{\alpha^{\prime}, \beta^{\prime}}$ and $\operatorname{otp}\left(C_{\alpha}\right) \leq \mu_{\beta^{\prime}}$. Then $C_{\alpha} \in M_{\alpha^{\prime}, \beta^{\prime}}$ and $C_{\alpha} \subseteq M_{\alpha^{\prime}, \beta^{\prime}}$. Also $\mu_{\beta} \in M_{\alpha^{\prime}, \beta^{\prime}}$ and $\mu_{\beta} \subseteq \mu_{\beta^{\prime}} \subseteq M_{\alpha^{\prime}, \beta^{\prime}}$. Hence it follows that $M_{\alpha, \beta}=\operatorname{sk}\left(\mu_{\beta} \cup C_{\alpha}\right) \subseteq M_{\alpha^{\prime}, \beta^{\prime}}$.]

Conversely, the existence of a $(\kappa, \mu)$-dominating matrix (over some/any $x)$ implies $\square_{\kappa, \mu}^{* * *}$ : 
THEOREM 8. Suppose that $\kappa$ is a regular cardinal and $\mu>\kappa$ is such that $\operatorname{cf}(\mu)<\kappa$. If there exists a $(\kappa, \mu)$-dominating matrix, then $\square_{\kappa, \mu}^{* * *}$ holds.

Proof. Let $\mu^{*}=\operatorname{cf}(\mu)$ and $\left(M_{\alpha, \beta}\right)_{\alpha<\mu^{+}, \beta<\mu^{*}}$ be a $(\kappa, \mu)$-dominating matrix. Let $M_{\alpha}=\bigcup_{\beta<\mu^{*}} M_{\alpha, \beta}$ for each $\alpha<\mu^{+}$. For

$$
X=\bigcup\left\{\left[M_{\alpha, \beta}\right]^{<\kappa} \cap M_{\alpha, \beta}: \alpha<\mu^{+}, \beta<\mu^{*}\right\},
$$

let $\left\{C_{\alpha+1}: \alpha<\mu^{+}\right\}$be an enumeration of $X$. Let

$$
\begin{aligned}
& D=\left\{\alpha<\mu^{+}: M_{\alpha} \cap \mu^{+}=\alpha,\left\{C_{\alpha^{\prime}+1}: \alpha^{\prime}<\alpha\right\} \supseteq\left[M_{\alpha^{\dagger}, \beta}\right]^{<\kappa} \cap M_{\alpha^{\dagger}, \beta}\right. \\
&\text { for every } \left.\alpha^{\dagger}<\alpha, \beta<\mu^{*}\right\} .
\end{aligned}
$$

By (j4), $D$ is a club subset of $\mu^{+}$. For $\alpha \in D$ with $\operatorname{cf}(\alpha) \geq \kappa$, let $C_{\alpha}=$ $M_{\alpha, \beta_{\alpha}} \cap \alpha$ where $\beta_{\alpha}<\mu^{+}$be such that $M_{\alpha, \beta_{\alpha}} \cap \alpha$ is cofinal in $\alpha$ (this is possible as $M_{\alpha} \cap \mu^{+}=\alpha$ and $\left.\mu^{*}<\kappa\right)$ and that $\left[M_{\alpha, \beta_{\alpha}}\right]^{<\kappa} \cap M_{\alpha, \beta_{\alpha}}$ is cofinal in $\left[M_{\alpha, \beta_{\alpha}}\right]^{<\kappa}$ (possible by $(\mathrm{j} 3)$ ). For $\alpha \in \operatorname{Lim}\left(\mu^{+}\right) \backslash\{\alpha \in D: \operatorname{cf}(\alpha) \geq \kappa\}$, let $C_{\alpha}$ be anything, say $C_{\alpha}=\emptyset$. We claim that $\left(C_{\alpha}\right)_{\alpha<\mu^{+}}$and $D$ as above satisfy the conditions in the definition of $\square_{\kappa, \mu}^{* * *}$ : (y1) is clear by definition of $C_{\alpha}$ 's. To show (y2), let $\alpha \in D$ be such that $\operatorname{cf}(\alpha) \geq \kappa$ and $x \in\left[C_{\alpha}\right]^{<\kappa}$. Then, by the choice of $\beta_{\alpha}$, there is $y \in[\alpha]^{<\kappa} \cap M_{\alpha, \beta_{\alpha}}$ such that $x \subseteq y$. By (j4), there are $\alpha^{\dagger}<\alpha$ and $\beta^{\dagger}<\mu^{*}$ such that $y \in M_{\alpha^{\dagger}, \beta^{\dagger}}$. By definition of $D$, it follows that $y=C_{\alpha^{\prime}+1}$ for some $\alpha^{\prime}<\alpha$. This shows that $[\alpha]^{<\kappa} \cap\left\{C_{\alpha^{\prime}}: \alpha^{\prime}<\alpha\right\}$ dominates $\left[C_{\alpha}\right]^{<\kappa}$. - Theorem 8

Thus, by Theorems 7 and 8 , if $\operatorname{cf}\left([\lambda]^{<\kappa}, \subseteq\right)=\lambda$ for cofinally many $\lambda<\mu$, $\square_{\kappa, \mu}^{* * *}$ is equivalent to the existence of a $(\kappa, \mu)$-dominating matrix. The assumption " $\operatorname{cf}\left([\lambda]^{<\kappa}, \subseteq\right)=\lambda$ for cofinally many $\lambda<\mu$ " cannot be removed from this equivalence theorem since $\square_{\kappa, \mu}^{* * *}$ implies this. However, using the following weakening of the notion of dominating matrix, we obtain a characterization of $\square_{\kappa, \mu}^{* * *}$ in ZFC: for a regular cardinal $\kappa$ and $\mu>\kappa$ such that $\mu^{*}=\operatorname{cf}(\mu)<\kappa$, let us call a matrix $\left(M_{\alpha, \beta}\right)_{\alpha<\mu^{+}, \beta<\mu^{*}}$ of elementary submodels of $\mathcal{H}(\chi)$ for a sufficiently large $\chi$ a weak $(\kappa, \mu)$-dominating matrix over $x$ if it satisfies (j1), (j2), (j4) for $M_{\alpha}=\bigcup_{\beta<\mu^{*}} M_{\alpha, \beta}, \alpha<\mu^{+}$, and

$\left(\mathrm{j} 3^{-}\right)$if $\alpha<\mu^{+}$is such that $\operatorname{cf}(\alpha) \geq \kappa$, then there is $\beta^{*}<\mu^{*}$ such that, for every $\beta^{*} \leq \beta<\mu^{*},\left[M_{\alpha, \beta}\right]^{<\kappa} \cap M_{\alpha}$ is cofinal in $\left(\left[M_{\alpha, \beta}\right]^{<\kappa}, \subseteq\right)$.

Since $\mu^{*}<\kappa$, the condition above is equivalent to:

$\left(\mathrm{j} 3^{*}\right)$ if $\alpha<\mu^{+}$is such that $\operatorname{cf}(\alpha) \geq \kappa$, then there is $\beta^{*}<\mu^{*}$ such that, for every $\beta^{*} \leq \beta<\mu^{*}$, there is $\beta^{\prime}<\mu^{*}$ such that $\left[M_{\alpha, \beta}\right]^{<\kappa} \cap M_{\alpha, \beta^{\prime}}$ is cofinal in $\left(\left[M_{\alpha, \beta}\right]^{<\kappa}, \subseteq\right)$.

TheOREM 9. Suppose that $\kappa$ is a regular cardinal and $\mu>\kappa$ is such that $\mu^{*}=\operatorname{cf}(\mu)<\kappa$. Then $\square_{\kappa, \mu}^{* * *}$ holds if and only if there is a weak $(\kappa, \mu)$ dominating matrix over some/any $x$. 
Proof. For the forward direction the proof is almost the same as that of Theorem 7 . We let $\left(\mu_{\beta}\right)_{\beta<\mu^{*}}$ be here merely an increasing sequence of regular cardinals with the limit $\mu$. Then $\left(M_{\alpha, \beta}\right)_{\alpha \in D, \beta<\mu^{*}}$ is constructed just as in the proof of Theorem 7 . Lemma 5 is then used to see that $\left(\mathrm{j} 3^{-}\right)$is satisfied by this matrix. For the converse, just the same proof as that of Theorem 8 will do.

Existence of a (weak) dominating matrix is not a theorem in ZFC: we show in Section 4 that Chang's Conjecture for $\aleph_{\omega}$ together with $2^{\aleph_{\omega}}=\aleph_{\omega+1}$ implies that there is no $\left(\aleph_{n}, \aleph_{\omega}\right)$-dominating matrix for any $n \geq 1$.

3. A characterization of the $\kappa$-Freese-Nation property. The following game over a partial ordering $P$ was considered in [6, 7]. Let $\mathcal{G}^{\kappa}(P)$ be the following game played by Players I and II: in a play in $\mathcal{G}^{\kappa}(P)$, Players I and II choose subsets $X_{\alpha}$ and $Y_{\alpha}$ of $P$ of cardinality less than $\kappa$ alternately for $\alpha<\kappa$ such that

$$
X_{0} \subseteq Y_{0} \subseteq X_{1} \subseteq Y_{1} \subseteq \ldots \subseteq X_{\alpha} \subseteq Y_{\alpha} \subseteq \ldots \subseteq X_{\beta} \subseteq Y_{\beta} \subseteq \ldots
$$

for $\alpha \leq \beta<\kappa$. Thus a play in $\mathcal{G}^{\kappa}(P)$ looks like

Player $I: X_{0}, X_{1}, \ldots, X_{\alpha}, \ldots$

Player $I I: Y_{0}, Y_{1}, \ldots, Y_{\alpha}, \ldots$

where $\alpha<\kappa$. Player II wins the play if $\bigcup_{\alpha<\kappa} X_{\alpha}=\bigcup_{\alpha<\kappa} Y_{\alpha}$ is a $\kappa$ subordering of $P$. Let us call a strategy $\tau$ for Player II simple if, in $\tau$, each $Y_{\alpha}$ is decided from the information of the set $X_{\alpha} \subseteq P$ alone (i.e. also independent of $\alpha$ ).

Another notion we need here is the following generalization of $V_{\kappa}$-likeness. Let $\kappa$ be regular and $\chi$ be sufficiently large. For $\mathcal{D} \subseteq\{M \prec \mathcal{H}(\chi)$ : $|M|<\kappa\}$, we say that $M \in[\mathcal{H}(\chi)]^{\kappa}$ is $\mathcal{D}$-approachable if there is an increasing sequence $\left(D_{\alpha}\right)_{\alpha<\kappa}$ of elements of $\mathcal{D}$ such that

(a) $D_{\alpha} \cup\left\{D_{\alpha}\right\} \subseteq D_{\alpha+1}$ for every $\alpha<\kappa$; and

(b) $M=\bigcup_{\alpha<\kappa} D_{\alpha}$.

Clearly $M \prec \mathcal{H}(\chi)$ is $V_{\kappa}$-like if and only if $M$ is $\mathcal{D}$-approachable for $\mathcal{D}=$ $\{M \prec \mathcal{H}(\chi):|M|<\kappa\}$.

A slightly weaker version of the following theorem was announced in [6]:

Theorem 10. Let $\kappa$ be a regular uncountable cardinal and $\kappa \leq \lambda$. Suppose that

(i) $\left([\mu]^{<\kappa}, \subseteq\right)$ has a cofinal subset of cardinality $\mu$ for every $\mu$ such that $\kappa<\mu<\lambda$ and $\operatorname{cf}(\mu) \geq \kappa$; and

(ii) $\square_{\kappa, \mu}^{* * *}$ holds for every $\mu$ such that $\kappa \leq \mu<\lambda$ and $\operatorname{cf}(\mu)<\kappa$.

Then, for a partial ordering $P$ of cardinality $\leq \lambda$, the following are equivalent: 
(1) P has the $\kappa-F N$;

(2) Player II has a simple winning strategy in $\mathcal{G}^{\kappa}(P)$;

(3) for some, or equivalently any sufficiently large $\chi$, and any $V_{\kappa}$-like $M \prec \mathcal{H}(\chi)$ with $P, \kappa \in M$, we have $P \cap M \leq_{\kappa} P$;

(4) for some, or equivalently any sufficiently large $\chi$, there is $\mathcal{D} \subseteq$ $[\mathcal{H}(\chi)]^{<\kappa}$ such that $\mathcal{D}$ is cofinal in $[\mathcal{H}(\chi)]^{<\kappa}$ and for any $\mathcal{D}$-approachable $M \subseteq \mathcal{H}(\chi)$, we have $P \cap M \leq_{\kappa} P$.

Note that $\neg 0^{\#}$ implies the conditions (i) and (ii). Also note that, for every $\lambda<\kappa^{+\omega}$, the condition (i) holds in ZFC. Hence the characterization above holds for partial orderings of cardinality $\leq \kappa^{+\omega}$ without any additional assumptions.

Proof (of Theorem 10). A proof of $(1) \Rightarrow(2) \Rightarrow(3)$ is given in [6]. For $(3) \Rightarrow(4)$, suppose that $P$ satisfies (3). Then $P$ together with $\mathcal{D}=\{M \prec$ $\mathcal{H}(\chi):|M|<\kappa, P, \kappa \in M\}$ satisfies (4). The proof of $(4) \Rightarrow(1)$ is done by induction on $\nu=|P| \leq \lambda$. If $\nu \leq \kappa$, then $P$ has the $\kappa$-FN by Lemma 1 . For $\nu>\kappa$, let $P$ and $\mathcal{D}$ be as in (4) and assume that $(4) \Rightarrow(1)$ holds for every partial ordering of cardinality $<\nu$. We need the following claims:

Claim 10.1. Let $\chi^{*}$ be sufficiently large above $\chi$. Suppose that $M$ is an elementary submodel of $\mathcal{H}\left(\chi^{*}\right)$ such that $P, \mathcal{H}(\chi), \mathcal{D} \in M, \kappa+1 \subseteq M$ and $[M]^{<\kappa} \cap M$ is cofinal in $[M]^{<\kappa}$ with respect to $\subseteq$. Then $P \cap M \leq_{\kappa} P$.

$\vdash$ Suppose not. Then there is $b \in P$ such that either

(a) $(P \cap M) \uparrow b$ has no cofinal subset of cardinality $<\kappa$; or

(b) $(P \cap M) \uparrow b$ has no coinitial subset of cardinality $<\kappa$.

To be definite, assume that we have case (a) - for case (b), just the same argument will do. We can construct an increasing sequence $\left(N_{\alpha}\right)_{\alpha<\kappa}$ of elements of $\mathcal{D}$ such that

(c) $N_{\alpha} \in M$ and $\left|N_{\alpha}\right|<\kappa$ for $\alpha<\kappa$ (since $\kappa+1 \subseteq M$, it follows that $\left.N_{\alpha} \subseteq M\right)$

(d) $N_{\alpha} \in N_{\alpha+1}$ for every $\alpha<\kappa$;

(e) $\left(P \cap N_{\alpha}\right)\left\lceil b\right.$ is not cofinal in $\left(P \cap N_{\alpha+1}\right) \mid b$ for every $\alpha<\kappa$.

Then $N=\bigcup_{\alpha<\kappa} N_{\alpha}$ is a $\mathcal{D}$-approachable elementary submodel of $\mathcal{H}(\chi)$ by (c) and (d). Hence, by (4), we have $P \cap M \leq_{\kappa} P$. But, by (e), $(P \cap N) \uparrow b$ has no cofinal subset of cardinality $<\kappa$. This is a contradiction.

To see that the construction of $N_{\gamma}$ is possible at a limit $\gamma<\kappa$, assume that $N_{\alpha}, \alpha<\gamma$, have been constructed in accordance with (c), (d) and (e). Let $N^{\prime}=\bigcup_{\alpha<\gamma} N_{\alpha}$. By (c), we have $N^{\prime} \subseteq M$ and $\left|N^{\prime}\right|<\kappa$. Since $[M]^{<\kappa} \cap M$ is cofinal in $[M]^{<\kappa}$, there is some $N^{\prime \prime} \in M$ such that $N^{\prime} \subseteq N^{\prime \prime} \subseteq \mathcal{H}(\chi)$ and $\left|N^{\prime \prime}\right|<\kappa$. Hence by elementarity of $M$ and by $\mathcal{D} \in M$ there is $N^{\prime \prime \prime} \in M \cap \mathcal{D}$ such that $N^{\prime \prime} \subseteq N^{\prime \prime \prime}$. Clearly, we may let $N_{\gamma}=N^{\prime \prime \prime}$. For the construction at 
a successor step, assume that $N_{\alpha}$ have been chosen in accordance with (c), (d) and (e). By assumption, there is $c \in(P \cap M)\left\lceil b\right.$ such that there is no $c^{\prime} \in$ $\left(P \cap N_{\alpha}\right)\left\lceil b\right.$ with $c \leq c^{\prime}$. By elementarity of $M$ there is $N^{*} \in M \cap \mathcal{D}$ such that $N_{\alpha} \cup\left\{N_{\alpha}, c\right\} \subseteq N^{*}$ and $\left|N^{*}\right|<\kappa$. Then $N_{\alpha+1}=N^{*}$ is as desired. $\dashv_{\text {Claim } 10.1}$

Claim 10.2. If $Q \leq_{\kappa} P$, then for every $\mathcal{D}$-approachable $M \prec \mathcal{H}(\chi)$ with $Q \in M$ we have $Q \cap M \leq_{\kappa} Q$. In particular, such a $Q$ also satisfies condition (4).

$\vdash$ Let $M \prec \mathcal{H}(\chi)$ be $\mathcal{D}$-approachable with $Q \in M$. By assumption, we have $P \cap M \leq_{\kappa} P$. By elementarity of $M$ and since $Q \in M$, we have $Q \cap M \leq_{\kappa} P \cap M$. It follows that $Q \cap M \leq_{\kappa} P$ and hence $Q \cap M \leq_{\kappa} Q$. Now, let $\mathcal{D}_{0}=\{M \in \mathcal{D}: Q \in M\}$. Then it is clear that $Q$ satisfies (4) with $\mathcal{D}_{0}$ in place of $\mathcal{D} . \dashv_{\text {Claim } 10.2}$

Now we are ready to prove the induction steps.

Case I: $\nu$ is a limit cardinal or $\nu=\mu^{+}$with $\operatorname{cf}(\mu) \geq \kappa$. Let $\nu^{*}=\operatorname{cf} \nu$. Then, by (i), we can find an increasing sequence $\left(M_{\alpha}\right)_{\alpha<\nu^{*}}$ of elementary submodels of $\mathcal{H}\left(\chi^{*}\right)$ such that, for every $\alpha<\nu^{*},\left|M_{\alpha}\right|<\nu$ and $M_{\alpha}$ satisfies the conditions in Claim 10.1; and $P \subseteq \bigcup_{\alpha<\nu^{*}} M_{\alpha}$. By Claim 10.1, $P \cap M_{\alpha} \leq_{\kappa}$ $P$ for every $\alpha<\nu^{*}$. For $\alpha<\nu^{*}$ let

$$
P_{\alpha}= \begin{cases}P \cap M_{\alpha} & \text { if } \alpha \text { is a successor, } \\ P \cap\left(\bigcup_{\beta<\alpha} M_{\beta}\right) & \text { otherwise. }\end{cases}
$$

Then $\left(P_{\alpha}\right)_{\alpha<\nu^{*}}$ is a continuously increasing sequence of suborderings of $P$ such that $\left|P_{\alpha}\right|<\nu$ for every $\alpha<\nu^{*}$ and $P=\bigcup_{\alpha<\nu^{*}} P_{\alpha}$. We also have $P_{\alpha} \leq_{\kappa} P$ for every $\alpha<\nu^{*}$ : for a successor $\alpha<\nu^{*}$ this is clear. If a limit $\alpha<\nu^{*}$ has cofinality $<\kappa$ then $P_{\alpha}$ can be represented as the union of an increasing sequence of $<\kappa$ many $\kappa$-subordering of $P$ and hence $P_{\alpha} \leq_{\kappa} P$. If $\alpha<\nu^{*}$ is a limit with cofinality $\geq \kappa$, then $P_{\alpha}=P \cap M$ where $M=\bigcup_{\beta<\alpha} M_{\beta}$. Now it is clear that $M$ satisfies the conditions in Claim 10.1. Hence we again obtain $P_{\alpha}=P \cap M \leq_{\kappa} P$. Now, by Claim 10.2, each of $P_{\alpha}, \alpha<\nu^{*}$, satisfies condition (4) and hence, by the induction hypothesis, has the $\kappa$-FN. Thus, by Lemma $2, P$ also has the $\kappa$-FN.

Case II: $\nu=\mu^{+}$with $\operatorname{cf}(\mu)<\kappa$. Let $\mu^{*}=\operatorname{cf}(\mu)$. Without loss of generality we may assume that the underlying set of $P$ is $\nu$. By Theorem 7 , there is a $(\kappa, \mu)$-dominating matrix $\left(M_{\alpha, \beta}\right)_{\alpha<\nu, \beta<\mu^{*}}$ over $(P, \mathcal{H}(\chi))$. For $\alpha<\nu$ and $\beta<\mu^{*}$, let $P_{\alpha, \beta}=P \cap M_{\alpha, \beta}$ and $P_{\alpha}=\bigcup_{\beta<\mu^{*}} P_{\alpha, \beta}$. By (j4), the sequence $\left(P_{\alpha}\right)_{\alpha<\nu}$ is continuously increasing and $\bigcup_{\alpha<\nu} P_{\alpha}=P$. $\left|P_{\alpha}\right| \leq \mu$ for every $\alpha<\nu$ by (j1).

Claim 10.3. $P_{\alpha} \leq_{\kappa} P$ for every $\alpha<\nu$.

$\vdash$ For $\alpha<\nu$ such that $\operatorname{cf}(\alpha) \geq \kappa$, we have $P_{\alpha, \beta} \leq_{\kappa} P$ for every sufficiently large $\beta<\mu^{*}$ by (j3) and Claim 10.1. Since $\mu^{*}<\kappa$, it follows that $P_{\alpha} \leq_{\kappa} P$. 
If $\operatorname{cf}(\alpha)<\kappa$, then, by the argument above, we have $P_{\alpha^{\prime}} \leq_{\kappa} P$ for every $\alpha^{\prime}<\alpha$ with $\operatorname{cf}\left(\alpha^{\prime}\right) \geq \kappa$. Since a $P_{\alpha}$ can be represented as the union of $<\kappa$ such $P_{\alpha^{\prime}}$ 's, it follows again that $P_{\alpha} \leq{ }_{\kappa} P$. $\dashv_{\text {Claim } 10.3}$

Now, by Claim 10.2, each of $P_{\alpha}, \alpha<\nu$, satisfies the condition of (4). Hence, by the induction hypothesis, they have the $\kappa$-FN. By Lemma 2 , it follows that $P$ also has the $\kappa$-FN.

Corollary 11. Suppose that $\kappa$ and $\lambda$ satisfy (i), (ii) in Theorem 10 and $2^{<\kappa}=\kappa$. Then:

(a) Every $\kappa$-cc complete Boolean algebra of cardinality $\leq \lambda$ has the $\kappa$-FN.

(b) For any $\mu$ such that $\mu^{<\kappa} \leq \lambda$, the partial ordering $\left([\mu]^{<\kappa}, \subseteq\right)$ has the $\kappa-F N$

Pr o of. Let $\chi$ be sufficiently large. For (a), let $B$ be a $\kappa$-cc complete Boolean algebra. We show that $B$ satisfies (3) of Theorem 10. Let $M \prec \mathcal{H}(\chi)$ be $V_{\kappa}$-like with $B, \kappa \in M$. By $2^{<\kappa}=\kappa$, Lemma 6 and by the remark after the lemma, we have $[M]^{<\kappa} \subseteq M$. Hence $B \cap M$ is a complete subalgebra of $B$. It follows that $B \cap M \leq_{\kappa} M$. For (b), let $M \prec \mathcal{H}(\chi)$ be $V_{\kappa}$-like with $\lambda, \kappa \in M$. Then as above we have $[M]^{<\kappa} \subseteq M$. Hence, letting $X=\lambda \cap M$, we have $[\lambda]^{<\kappa} \cap M=[X]^{<\kappa} \leq_{\kappa}[\lambda]^{<\kappa}$. Corollary 11

4. Chang's Conjecture for $\aleph_{\omega}$. Recall that $(\kappa, \lambda) \rightarrow(\mu, \nu)$ is the following assertion:

For any structure $\mathcal{A}=(A, U, \ldots)$ of countable signature with $|A|=$ $\kappa, U \subseteq A$ and $|U|=\lambda$, there is an elementary substructure $\mathcal{A}^{\prime}=$ $\left(A^{\prime}, U^{\prime}, \ldots\right)$ of $\mathcal{A}$ such that $\left|A^{\prime}\right|=\mu$ and $\left|U^{\prime}\right|=\nu$.

In [13], a model of ZFC + GCH + Chang's Conjecture for $\aleph_{\omega}$, i.e. $\left(\aleph_{\omega+1}, \aleph_{\omega}\right)$ $\rightarrow\left(\aleph_{1}, \aleph_{0}\right)$, is constructed starting from a model with a cardinal having a property slightly stronger than huge. The following theorem together with Corollary 11 shows that the $\aleph_{1}$-FN of the partial ordering $\left(\left[\aleph_{\omega}\right]^{\aleph_{0}}, \subseteq\right)$ is independent of ZFC (or even of ZFC $+\mathrm{GCH}$ ).

TheOREM 12. Suppose that $\left(\aleph_{\omega}\right)^{\aleph_{0}}=\aleph_{\omega+1}$ and $\left(\aleph_{\omega+1}, \aleph_{\omega}\right) \rightarrow\left(\aleph_{1}, \aleph_{0}\right)$. Then $\left(\left[\aleph_{\omega}\right]^{\aleph_{0}}, \subseteq\right)$ does not have the $\aleph_{1}-F N$.

Proof. Assume to the contrary that there is an $\aleph_{1}$-FN mapping $F$ : $\left[\aleph_{\omega}\right]^{\aleph_{0}} \rightarrow\left[\left[\aleph_{\omega}\right]^{\aleph_{0}}\right]^{\aleph_{0}}$. Fix an enumeration $\left(b_{\alpha}\right)_{\alpha<\aleph_{\omega+1}}$ of $\left[\aleph_{\omega}\right]^{\aleph_{0}}$ and consider the structure

$$
\mathcal{A}=\left(\aleph_{\omega+1}, \aleph_{\omega}, \leq, E, f, g, h\right)
$$

where

(1) $\leq$ is the canonical ordering on $\aleph_{\omega+1}$;

(2) $E=\left\{(\alpha, \beta): \alpha \in \aleph_{\omega}, \beta \in \aleph_{\omega+1}, \alpha \in b_{\beta}\right\}$; 
(3) $f: \aleph_{\omega+1} \times \aleph_{\omega+1} \rightarrow \aleph_{\omega+1}$ is such that, for each $\alpha \in \aleph_{\omega+1}, F\left(b_{\alpha}\right)=$ $\left\{b_{f(\alpha, n)}: n \in \omega\right\}$

(4) $g: \aleph_{\omega+1} \times \aleph_{\omega+1} \rightarrow \aleph_{\omega}$ is such that, for each $\alpha \in \aleph_{\omega+1}, g(\alpha, \cdot) \mid \alpha$ is an injective mapping from $\alpha$ to $\aleph_{\omega}$; and

(5) $h: \aleph_{\omega+1} \times \aleph_{\omega+1} \times \aleph_{\omega+1} \rightarrow \omega+1$ is such that for each $\alpha, \beta \in \aleph_{\omega+1}$, $h(\alpha, \beta, \cdot)\left\lceil\left(b_{\alpha} \cap b_{\beta}\right)\right.$ is injective.

Note that, by (5) and since $\omega$ is definable in $\mathcal{A}$, we can express " $b_{\alpha} \cap b_{\beta}$ is finite" as a formula $\varphi(\alpha, \beta)$ in the language of $\mathcal{A}$. Now, applying Chang's Conjecture for $\mathcal{A}$ with $A=\aleph_{\omega+1}$ and $U=\aleph_{\omega}$, we obtain an elementary substructure $\mathcal{A}^{\prime}=\left(A^{\prime}, U^{\prime}, \leq^{\prime}, E^{\prime}, f^{\prime}, g^{\prime}, h^{\prime}\right)$ of $\mathcal{A}$ such that $\left|A^{\prime}\right|=\aleph_{1}$ and $\left|U^{\prime}\right|=\aleph_{0}$.

Claim 12.1. otp $\left(A^{\prime}\right)=\omega_{1}$.

$\vdash$ By (4) and elementarity of $\mathcal{A}^{\prime}$, every initial segment of $A^{\prime}$ can be mapped into $U^{\prime}$ injectively and hence is countable. Since $\left|A^{\prime}\right|=\aleph_{1}$, it follows that $\operatorname{otp}\left(A^{\prime}\right)=\omega_{1} \cdot \dashv_{\text {Claim } 12.1}$

Claim 12.2. For any $\alpha<\aleph_{\omega+1}$, there is $\gamma<\aleph_{\omega+1}$ such that $b_{\beta} \cap b_{\gamma}$ is finite for every $\beta<\alpha$.

$\vdash$ Since $|\alpha| \leq \aleph_{\omega}$, we can find a partition $\left(I_{n}\right)_{n \in \omega}$ of $\alpha$ such that $\left|I_{n}\right|<\aleph_{\omega}$ for every $n<\omega$. For $n<\omega$, let $\eta_{n}=\min \left(\aleph_{\omega} \backslash \bigcup\left\{b_{\xi}: \xi \in \bigcup_{m \leq n} I_{m}\right\}\right)$. Let $z=\left\{\eta_{n}: n \in \omega\right\}$ and $\gamma<\aleph_{\omega+1}$ be such that $b_{\gamma}=z$. For any $\beta<\alpha$, if $\beta \in I_{m_{0}}$ for some $m_{0}<\omega$, then $b_{\beta} \cap b_{\gamma} \subseteq\left\{\eta_{n}: n<m_{0}\right\}$. Thus this $\gamma$ is as desired. $\dashv_{\text {Claim } 12.2}$

Claim 12.3. For any countable $I \subseteq A^{\prime}$, there is $\gamma \in A^{\prime}$ such that $b_{\beta} \cap b_{\gamma}$ is finite for every $\beta \in I$.

$\vdash$ By Claim 12.1, there is $\alpha \in A^{\prime}$ such that $I \subseteq \alpha$. By elementarity of $\mathcal{A}^{\prime}$, the formula with the parameter $\alpha$ expressing the assertion of Claim 12.2 for this $\alpha$ holds in $\mathcal{A}^{\prime}$. Hence there is some $\gamma \in A^{\prime}$ such that $b_{\beta} \cap b_{\gamma}$ is finite for every $\beta \in A^{\prime} \cap \alpha . \dashv_{\text {Claim } 12.3}$

Let

$$
I=\left\{\xi \in A^{\prime}: b_{\xi} \in F\left(U^{\prime}\right)\right\} .
$$

Then $I$ is countable. Hence, by Claim 12.3 , there is $\gamma \in A^{\prime}$ such that $b_{\beta} \cap b_{\gamma}$ is finite for every $\beta \in I$. As $b_{\gamma} \subseteq U^{\prime}$ (this holds in virtue of $h(\gamma, \gamma, \cdot)$ ), there is $b \in F\left(b_{\gamma}\right) \cap F\left(U^{\prime}\right)$ such that $b_{\gamma} \subseteq b \subseteq U^{\prime}$. Let $b=b_{\xi_{0}}$. Then $\xi_{0} \in I$ and $\left|b_{\gamma} \cap b_{\xi_{0}}\right|=\left|b_{\gamma}\right|=\aleph_{0}$. This is a contradiction to the choice of $\gamma$. Theorem 12

We do not know if the assumption of Theorem 12 yields a counterexample to Corollary 11(a) for $\kappa=\aleph_{1}$. Or, more generally:

Problem 2. Is there a model of $\mathrm{ZFC}+\mathrm{GCH}$ where some ccc complete Boolean algebra does not have the $\aleph_{1}-F N$ ? 
Of course, we need the consistency strength of some large cardinal to obtain such a model by Corollary 11 .

The following corollary slightly improves Theorem 4.1 of [3].

Corollary 13. Suppose that $\left(\aleph_{\omega}\right)^{\aleph_{0}}=\aleph_{\omega+1}$ and $\left(\aleph_{\omega+1}, \aleph_{\omega}\right) \rightarrow\left(\aleph_{1}, \aleph_{0}\right)$. Then the equivalence of the assertions in Theorem 10 fails. Hence we have $\neg \square_{\aleph_{1}, \aleph_{\omega}}^{* * *}$ under these assumptions.

Proof. By Theorem 12 and Corollary 11(b). orollary 13

Similarly we can prove $\neg \square_{\aleph_{n}, \aleph_{\omega}}^{* * *}$ for every $n \in \omega$ under the assumption of $2^{\aleph_{\omega}}=\aleph_{\omega+1}$ and $\left(\aleph_{\omega+1}, \aleph_{\omega}\right) \rightarrow\left(\aleph_{1}, \aleph_{0}\right)$.

5. Cohen models. Let $V$ be our ground model and let $G$ be a $V$ generic filter over $P=\operatorname{Fn}(\tau, 2)$ for some $\tau$. Suppose that $B$ is a ccc complete Boolean algebra in $V[G]$. Without loss of generality we may assume that the underlying set of $B$ is a set $X$ in $V . B$ is said to be tame if there is a $P$-name $\dot{\leq}$ of partial ordering of $B$ and a mapping $t: X \rightarrow[\tau]^{\aleph_{0}}$ in $V$ such that, for every $p \in P$ and $x, y \in X$, if $p \Vdash_{P}$ " $x \dot{\leq} y$ ", then $p\left\lceil(t(x) \cup t(y)) \Vdash_{P}\right.$ " $x \dot{\leq} y$ ". A lot of "natural" ccc complete Boolean algebras in $V[G]$ are contained in the class of tame Boolean algebras:

Lemma 14. Let $G$ be as above. Suppose that $V[G] \vDash " B$ is a ccc complete Boolean algebra" and either:

(i) there is a Boolean algebra $B^{\prime}$ in $V$ such that $B^{\prime}$ is dense subalgebra of $B$ in $V[G]$; or

(ii) $B$ is the completion of a Suslin forcing in $V[G]$.

Then $B$ is tame.

For Suslin forcing, see e.g. [1].

Theorem 15. Let $P, G$ be as above and $\lambda$ an infinite cardinal. Assume that, in $V$,

(i) $\mu^{\aleph_{0}}=\mu$ for every regular uncountable $\mu$ such that $\mu<\lambda$; and

(ii) $\square_{\aleph_{1}, \mu}^{* * *}$ holds for every $\mu$ such that $\aleph_{0}<\mu<\lambda$ and $\operatorname{cf}(\mu)=\omega$.

Then for any tame ccc complete Boolean algebra $B$ in $V[G]$ of cardinality $\leq \lambda$ we have $V[G] \vDash$ "B has the $\aleph_{1}-F N$ ".

Proof. Let $X, \dot{\leq}$ and $t$ be as in the definition of tameness for $B$ above. We may assume $\Vdash_{P}$ " $X$ is the underlying set of $\dot{B}$ " and $\Vdash_{P}$ " $\dot{B}$ is a ccc complete Boolean algebra" where $\dot{B}$ is a $P$-name for $B$. Let $\chi$ be sufficiently large. The following is the key lemma to the proof:

Claim 15.1. Suppose that $M \prec \mathcal{H}(\chi)$ is such that $\tau, X, \dot{\leq}, t \in M$ and $[M]^{\aleph_{0}} \subseteq M$. Let $I=\tau \cap M, P^{\prime}=\operatorname{Fn}(I, 2), G^{\prime}=G \cap P^{\prime}, X^{\prime}=X \cap M$ and $\dot{\leq}^{\prime}=\dot{\leq} \cap M$. Then 
(a) $\dot{\leq}^{\prime}$ is a $P^{\prime}$-name, $\Vdash_{P}$ " $B^{\prime}=\left(X^{\prime}, \dot{\leq}^{\prime}\right)$ is a ccc complete Boolean algebra", $B^{\prime}$ is tame (in $\left.V\left[G^{\prime}\right]\right)$ and the infinite sum $\Sigma^{B}$ in $V[G]$ is an extension of the infinite sum $\Sigma^{B^{\prime}}$ in $V\left[G^{\prime}\right]$.

(b) $\Vdash_{P}$ " $\left(X^{\prime}, \dot{\leq}^{\prime}\right) \leq_{\sigma}(X, \dot{\leq})$ ".

$\vdash$ (a) It is easy to see that $\Vdash_{P^{\prime}}$ " $\left(X^{\prime}, \dot{\leq}^{\prime}\right)$ is a Boolean algebra" and $\Vdash_{P}$ " $\left(X^{\prime}, \dot{\leq}^{\prime}\right)$ is a subalgebra of $(X, \dot{\leq})$ ". Since $\Vdash_{P}$ " $(X, \dot{\leq})$ has the ccc", it follows that $\Vdash_{P^{\prime}}$ " $\left(X^{\prime}, \dot{\leq}^{\prime}\right)$ has the ccc". By elementarity of $M$, it is also easy to see that $t\left\lceil X^{\prime}\right.$ witnesses the tameness of $B^{\prime}$ in $V\left[G^{\prime}\right]$. To see that $\Vdash_{P^{\prime}}$ " $\left(X^{\prime}, \dot{\leq}^{\prime}\right)$ is complete" it is enough to see that every countable subset of $B^{\prime}$ has its supremum in $V\left[G^{\prime}\right]$. Let $\dot{C}$ be a $P^{\prime}$-name of countable subset of $X^{\prime}$. Without loss of generality, we may assume that $\dot{C}$ is countable and consists of sets of the form $(p, \check{x})$ where $p \in P^{\prime}$ and $x \in X^{\prime}$. Since $[M]^{\aleph_{0}} \subseteq M$, $\dot{C} \in M$. Clearly, we have $M \vDash " \dot{C}$ is a $P$-name of countable subset of $X$ ". Hence, $M \vDash " \exists p \in P \exists y \in X\left(p \Vdash_{P}\right.$ " $\Sigma \dot{C}=y$ ")". Let $p \in P$ and $y \in X$ be such elements of $M$. Then $p \in P^{\prime}$ and $y \in X^{\prime}$. By elementarity of $M$, we have $p \Vdash_{P}$ " $\Sigma^{B} \dot{C}=y$ ". On the other hand, from $M \vDash " p \Vdash_{P}$ " $\Sigma \dot{C}=y$ " " it follows that $p \Vdash_{P^{\prime}} " \Sigma^{B^{\prime}} \dot{C}=y$ ".

(b) By assumption, for $x \in X$ and $y \in X^{\prime}$, we have $y \leq x$ in $V[G]$ if and only if there is $p \in G$ with $\operatorname{dom}(p) \subseteq t(x) \cup t(y)$ such that $p \Vdash_{P}$ " $y \dot{\leq} x$ ". For $q \in G \cap \operatorname{Fn}(t(y), 2)$, the set $U_{q}=\left\{y \in X^{\prime}: \exists p \in G^{\prime}\left(p \cup q \Vdash_{P}\right.\right.$ " $y \dot{\leq} x$ ") $)$ is in $V\left[G^{\prime}\right]$. Hence, by (a), $\Sigma^{B} U_{q}$ is an element in $B^{\prime}$. Since $X^{\prime}$ is closed under $t$, it follows that $\left\{\Sigma^{B} U_{q}: q \in G \cap \operatorname{Fn}(t(y), 2)\right\}$ is cofinal in $B^{\prime}\left\lceil y . \dashv_{\text {Claim } 15.1}\right.$

Now, let $\nu=|X|$. Without loss of generality, we may assume that $X=\nu$. We show by induction on $\nu$ that $\Vdash_{P}$ " $\dot{B}$ has the $\aleph_{1}$-FN". For $\nu \leq \aleph_{1}$ the assertion follows from Lemma 1 (applied in $V^{P}$ ). In the induction steps, we mimic the proof of Theorem 10. Let $\chi$ be sufficiently large.

Case I: $\nu$ is a limit cardinal or $\nu=\mu^{+}$for some $\mu$ with $\operatorname{cf}(\mu) \geq \omega_{1}$. By (i), we can construct a continuously increasing sequence $\left(M_{\alpha}\right)_{\alpha<\nu}$ of elementary submodels of $\mathcal{H}(\chi)$ such that

(0) $\tau, \nu, \dot{\leq}, t \in M_{0}$;

(1) $\left|M_{\alpha}\right|<\nu$ for every $\alpha<\nu$;

(2) $\left[M_{\alpha+1}\right]^{\aleph_{0}} \subseteq M_{\alpha+1}$ for every $\alpha<\nu$ (note that it follows that the inclusion also holds for every limit $<\nu$ of cofinality $\geq \omega_{1}$ ); and

(3) $\nu \subseteq \bigcup_{\alpha<\nu} M_{\alpha}$.

For each $\alpha<\nu$, let $X_{\alpha}=X \cap M_{\alpha}$ and $\dot{\leq}^{\alpha}=\dot{\leq} \cap M_{\alpha}$ and let $\dot{B}_{\alpha}$ be the $P$-name corresponding to $\left(X_{\alpha}, \dot{\leq}^{\alpha}\right)$. By Claim 15.1, we have $\Vdash_{P}$ " $\dot{B}_{\alpha}$ is a ccc complete Boolean algebra and $\dot{B}_{\alpha} \leq_{\aleph_{1}} \dot{B}$ ", for all $\alpha<\nu$ such that either $\alpha$ is a successor or of cofinality $\geq \omega_{1}$. By the induction hypothesis, we have $\Vdash_{P}$ " $\dot{B}_{\alpha}$ has the $\aleph_{1}$-FN" for such $\alpha$ 's. Hence by Lemma 2 
and the remark after the lemma (applied in $V^{P}$ ) it follows that $\Vdash_{P}$ " $\dot{B}$ has the $\aleph_{1}-\mathrm{FN} "$.

Case II: $\nu=\mu^{+}$for a $\mu$ with $\operatorname{cf}(\mu)=\omega$. By (ii), there is an $\left(\aleph_{1}, \mu\right)$ dominating matrix $\left(M_{\alpha, n}\right)_{\alpha<\nu, n<\omega}$ over $(\tau, \nu, \dot{\leq}, t)$. For $\alpha<\nu$, let $M_{\alpha}=$ $\bigcup_{n<\omega} M_{\alpha, n}$. For $\alpha<\nu$ and $n<\omega$, let $X_{\alpha, n}=X \cap M_{\alpha, n}, \dot{\leq}^{\alpha, n}=\dot{\leq} \cap$ $M_{\alpha, n}$ and $\dot{B}_{\alpha, n}$ be the $P$-name corresponding to $\left(X_{\alpha, n}, \dot{\leq}^{\alpha, n}\right)$. Likewise, let $X_{\alpha}=X \cap M_{\alpha}, \dot{\leq}^{\alpha}=\dot{\leq} \cap M_{\alpha}$ and $\dot{B}_{\alpha}$ be the $P$-name corresponding to $\left(X_{\alpha}, \dot{\leq}^{\alpha}\right)$. Then we have $X_{\alpha}=\bigcup_{n<\omega} X_{\alpha, n}, \dot{\leq}^{\alpha}=\bigcup_{n<\omega} \dot{\leq}^{\alpha, n}$ and $\Vdash_{P}$ " $\dot{B}_{\alpha}=\bigcup_{n<\omega} \dot{B}_{\alpha, n}$ ". By Lemma 6 and (i), (j3') holds for the dominating matrix. Hence, by Claim 15.1, we have $\Vdash_{P} " \dot{B}_{\alpha, n} \leq_{\aleph_{1}} \dot{B}_{\alpha}$ and $\dot{B}_{\alpha, n}$ is a ccc complete Boolean algebra" for every $\alpha<\nu$ with $\operatorname{cf}(\alpha)>\omega$ and every sufficiently large $n<\omega$. By the induction hypothesis, it follows that $\Vdash_{P}$ " $\dot{B}_{\alpha, n}$ has the $\aleph_{1}-\mathrm{FN}$ " for such $\alpha$ and $n$. By Lemma $2\left(\right.$ applied in $\left.V^{P}\right)$ it follows that $\Vdash_{P}$ " $\dot{B}_{\alpha}$ has the $\aleph_{1}$-FN" for every $\alpha<\nu$ with $\operatorname{cf}(\alpha)>\omega$. Hence again by Lemma 2 and the remark after it (applied in $V^{P}$ ) we conclude that $\Vdash_{P}$ " $\dot{B}$ has the $\aleph_{1}$-FN". - Theorem 15

COROllary 16. Suppose that $V=L$ holds and let $G$ be $V$-generic over $P=\operatorname{Fn}(\tau, 2)$ for some $\tau$. Then (among others) the following ccc complete Boolean algebras have the $\aleph_{1}-F N: \mathcal{C}_{\kappa}(\cong$ the completion of $\operatorname{Fn}(\kappa, 2))$ for any $\kappa ; \mathcal{P}(\omega)$ (hence also $\mathcal{P}(\omega) /$ fin); Borel $(\mathbb{R}) /$ Null-sets.

In connection with Theorem 15, we would like to mention the following open problems:

Problem 3. Assume that $V[G]$ is a model obtained by adding Cohen reals to a model of $\mathrm{ZFC}+\mathrm{CH}$. Is it true that $\mathcal{P}(\omega)$ has the $\aleph_{1}-F N$ in $V[G]$ ?

By Theorem 15, the answer to this question is positive if the number of Cohen reals added is less than $\aleph_{\omega}$.

Problem 4. Does Theorem 15 hold also without the assumption of tameness?

Or, more generally:

Problem 5. Are the following equivalent?

(i) $\mathcal{P}(\omega)$ has the $\aleph_{1}-F N$;

(ii) every ccc complete Boolean algebra has the $\aleph_{1}-F N$.

6. Lusin gap. For a regular $\kappa$, an almost disjoint family $\mathcal{A} \subseteq[\omega]^{\aleph_{0}}$ is said to be a $\kappa$-Lusin gap if $|\mathcal{A}|=\kappa$ and for any $x \in[\omega]^{\aleph_{0}}$ either $\left|\left\{a \in \mathcal{A}:|a \backslash x|<\aleph_{0}\right\}\right|<\kappa$ or $\left|\left\{a \in \mathcal{A}:|a \cap x|<\aleph_{0}\right\}\right|<\kappa$. 
Theorem 17. Assume that $\mathcal{P}(\omega)$ has the $\aleph_{1}-F N$. Then there is no $\aleph_{2}$ Lusin gap.

Pr o o f. Let $f: \mathcal{P}(\omega) \rightarrow[\mathcal{P}(\omega)]^{\aleph_{0}}$ be an $\aleph_{1}-\mathrm{FN}$ mapping. We may assume that $f(a)=f(b)=f(\omega \backslash b)$ for all $a, b \in \mathcal{P}(\omega)$ with $a={ }^{*} b$. Thus $x \subseteq^{*} y$ implies that there is $z \in f(x) \cap f(y)$ such that $x \subseteq^{*} z \subseteq^{*} y$, and $|x \cap y|<\aleph_{0}$ implies that there is $z \in f(x) \cap f(y)$ such that $x \subseteq^{*} z$ and $|z \cap y|<\aleph_{0}$.

Suppose that $\mathcal{A}=\left\{a_{\alpha}: \alpha<\omega_{2}\right\}$ is an almost disjoint family of subsets of $\omega$. We show that $\mathcal{A}$ is not an $\aleph_{2}$-Lusin gap. Let $\chi$ be a sufficiently large regular cardinal and consider the model $\mathcal{H}=(\mathcal{H}(\chi), \in, \unlhd)$ where $\unlhd$ is any well-ordering on $\mathcal{H}$. Let $N$ be an elementary submodel of $\mathcal{H}$ such that $\mathcal{A}, f \in N, N \cap \omega_{2} \in \omega_{2}$ and $\operatorname{cf}(\delta)=\omega_{1}$ for $\delta=N \cap \omega_{2}$. For $\alpha \in N$ we have $\left|a_{\alpha} \cap a_{\delta}\right|<\aleph_{0}$ and hence $a_{\alpha} \subseteq^{*}\left(\omega \backslash a_{\delta}\right)$. Thus there is $b_{\alpha} \in f\left(a_{\alpha}\right) \cap f\left(a_{\delta}\right)$ such that $a_{\alpha} \subseteq^{*} b_{\alpha} \subseteq^{*} \omega \backslash a_{\delta}$. Since $f\left(a_{\delta}\right)$ is countable and $\operatorname{cf}(\delta)=\omega_{1}$ there is $b \in f\left(a_{\delta}\right)$ such that $I=\left\{\alpha<\delta: b_{\alpha}=b\right\}$ is cofinal in $\delta$. We show that $b$ witnesses that $\mathcal{A}$ is not an $\aleph_{2}$-Lusin gap, i.e., $J=\left\{\alpha<\omega_{2}: a_{\alpha} \subseteq^{*} b\right\}$ and $K=\left\{\alpha<\omega_{2}:\left|b \cap a_{\alpha}\right|<\aleph_{0}\right\}$ both have cardinality $\aleph_{2}$.

CLAIM 17.1. $|J|=\aleph_{2}$.

$\vdash$ First note that $b \in N$ since $b \in f\left(a_{\alpha}\right)$ for any $\alpha \in I \subseteq N$. Hence $J \in N$ and $I \subseteq J$. Since $I$ is cofinal in $N \cap \omega_{2}$, we have $N \vDash$ " $J$ is cofinal in $\omega_{2}$ ". By elementarity it follows that $\mathcal{H} \vDash " J$ is cofinal in $\omega_{2}$ ". Hence $J$ is really cofinal in $\omega_{2} . \dashv_{\text {Claim } 17.1}$

Claim 17.2. $|K|=\aleph_{2}$.

$\vdash$ Since $b \in N$ it follows that $K \in N$. For $\beta \in N \cap \omega_{2}=\delta$, we have $\mathcal{H} \vDash " \delta \in K \wedge \beta<\delta$ ". Hence $\mathcal{H} \vDash " K \nsubseteq \not \beta$ " and $N \vDash " K \nsubseteq \beta$ " by elementarity. It follows that $N \vDash " K$ is unbounded in $\omega_{2}$ ". Hence, again by elementarity, $\mathcal{H} \vDash$ " $K$ is unbounded in $\omega_{2}$ ". Thus $K$ is really unbounded in $\omega_{2} \cdot \dashv$ Claim 17.2 -Theorem 17

Corollary $18 . \mathbf{b}=\aleph_{1}$ or even the statement " $\mathcal{P}(\omega)$ does not contain any strictly increasing $\subseteq{ }^{*}$-chain of length $\omega_{2}$ " does not imply that $\mathcal{P}(\omega)$ has the $\aleph_{1}-F N$.

Pro of. Suppose that our ground model $V$ satisfies the CH. Koppelberg and Shelah [11] proved that the forcing with $\operatorname{Fn}\left(\omega_{2}, 2\right)$ can be represented as a two-step iteration $A * \dot{B}$ where $\Vdash_{A}$ " $\mathcal{P}(\omega)$ contains an $\aleph_{2}$-Lusin gap". Thus, by Theorem 17 , we have $\Vdash_{A}$ " $\mathcal{P}(\omega)$ does not have the $\aleph_{1}$-FN". On the other hand, we have $\Vdash_{A * \dot{B}}$ "P $\mathcal{P}(\omega)$ does have the $\aleph_{1}$-FN" by Theorem 15. Hence $\Vdash_{A * \dot{B}}$ "there is no strictly increasing $\subseteq^{*}$-chain in $\mathcal{P}(\omega)$ of length $\omega_{2}$ ". It follows that $\Vdash_{A}$ "there is no strictly increasing $\subseteq^{*}$-chain in $\mathcal{P}(\omega)$ of length $\omega_{2} "$. - Corollary 18 


\section{References}

[1] T. Bartoszyński and H. Judah, Set Theory: on the structure of the real line, A K Peters, 1995.

[2] S. Ben-David and M. Magidor, The weak $\square$ is really weaker than full $\square$, J. Symbolic Logic 51 (1986), 1029-1033.

[3] M. Foreman and M. Magidor, A very weak square principle, preprint.

[4] M. Foreman, M. Magidor and S. Shelah, Martin's maximum, saturated ideals, and non-regular ultrafilters I, Ann. of Math. (2) 127 (1988), 1-47.

[5] R. Freese and J. B. Nation, Projective lattices, Pacific J. Math. 75 (1978), 93-106.

[6] S. Fuchino, S. Koppelberg and S. Shelah, Partial orderings with the weak Freese-Nation property, Ann. Pure Appl. Logic 80 (1996), 35-54.

[7] —, - - - A game on partial orderings, Topology Appl. 74 (1996), 141-148.

[8] L. Heindorf and L. B. Shapiro, Nearly Projective Boolean Algebras, Lecture Notes in Math. 1596, Springer, 1994.

[9] R. Jensen, The fine structure of the constructible hierarchy, Ann. Math. Logic 4 (1972), 229-308.

[10] S. Koppelberg, Applications of $\sigma$-filtered Boolean algebras, preprint.

[11] S. Koppelberg and S. Shelah, Subalgebras of the Cohen algebra do not have to be Cohen, preprint.

[12] K. Kunen, Set Theory, North-Holland, 1980.

[13] J.-P. Levinski, M. Magidor and S. Shelah, On Chang's conjecture for $\aleph_{\omega}$, Israel J. Math. 69 (1990), 161-172.

Institut für Mathematik II

Freie Universität Berlin

14195 Berlin, Germany

E-mail: fuchino@math.fu-berlin.de

Present address:

Department of Computer Sciences

Kitami Institute of Technology

Kitami Hokkaido 090, Japan

E-mail: fuchino@info.kitami-it.ac.jp
Mathematical Institute Hungarian Academy of Sciences Budapest, Hungary E-mail: soukup@math-inst.hu

Received 29 April 1996; in revised form 24 May 1996 